\title{
Chronic Fluoxetine Treatment Increases the Expression of PSA-NCAM in the Medial Prefrontal Cortex
}

\author{
Emilio Varea', José Miguel Blasco-lbáñez', María Ángeles Gómez-Climent', Esther Castillo-Gómez', \\ Carlos Crespo', Francisco José Martínez-Guijarro' and Juan Nácher*,' \\ 'Cell Biology Department, Neurobiology Unit and Program in Basic and Applied Neurosciences, Universitat de València, Burjassot, Spain
}

\begin{abstract}
Recent hypotheses suggest that changes in neuronal structure and connectivity may underlie the etiology of depression. The medial prefrontal cortex (mPFC) is affected by depression and shows neuronal remodeling during adulthood. This plasticity may be mediated by the polysialylated form of the neural cell adhesion molecule (PSA-NCAM), which is intensely expressed in the adult mPFC. As the expression of PSA-NCAM is increased by serotonin in other cerebral regions, antidepressants acting on serotonin reuptake may influence PSA-NCAM expression and thus counteract the effects of depression by modulating neuronal structural plasticity. Using immunohistochemistry, we have studied the relationship between serotoninergic fibers and PSA-NCAM expressing neurons in the adult rat $\mathrm{mPFC}$ and the expression of serotonin receptors in these cells. The effects of fluoxetine treatment for I 4 days on mPFC PSA-NCAM expression have also been analyzed. Although serotoninergic fibers usually do not contact PSA-NCAM immunoreactive neurons, most of these cells express 5-HT3 receptors. In general, chronic fluoxetine treatment induces significant increases in the number of PSA-NCAM immunoreactive neurons and in neuropil immunostaining and coadministration of the 5-HT3 antagonist ondansetron blocks the effects of fluoxetine on PSA-NCAM expression. These results indicate that fluoxetine, acting through 5-HT3 receptors, can modulate PSA-NCAM expression in the mPFC. This modulation may mediate the structural plasticity of this cortical region and opens new perspectives on the study of the molecular bases of depression.

Neuropsychopharmacology (2007) 32, 803-812. doi:10.1038/sj.npp. I301 I83; published online 9 August 2006
\end{abstract}

Keywords: serotonin; depression; interneuron; 5-HT receptor; structural plasticity; ondansetron

\section{INTRODUCTION}

The cellular bases of depression are not yet well understood, although recent hypotheses suggest that it may be caused by dysfunction of the normal mechanisms underlying the plasticity of neuronal networks (Castren, 2005; Duman et al, 1999). In fact, antidepressant drugs may act by normalizing this impairment (Duman et al, 1999; Manji et al, 2000, 2001; Manji and Duman, 2001). Structural plastic processes, such as dendritic or spine remodeling, have been observed in several regions of the adult CNS, specially as a response to aversive experiences (McEwen, 2000), or as a consequence of several learning paradigms (Muller et al, 2002). Interestingly, some of the regions displaying this plasticity are those affected by depression, such as the amygdala, the hippocampus, the basal ganglia, and several regions of the medial

\footnotetext{
*Correspondence: Dr J Nácher, Cell Biology Department, Neurobiology Unit and Program in Basic and Applied Neurosciences, Universitat de València, Dr Moliner, 50, Burjassot 46100, Spain, Tel: + 3496354 324I, Fax: + 3496354 324I, E-mail: nacher@uv.es

Received 2 December 2005; revised 4 May 2006; accepted 4 June 2006

Online publication: 13 July 2006 at http://www.acnp.org/citations/ Npp07| 3060507/2/default.pdf
}

prefrontal cortex (mPFC) (Bearden et al, 2001; Drevets et al, 1997; Fava and Kendler, 2000; Strakowski et al, 2000).

Brain imaging studies have demonstrated a reduction in blood flow, volume, number of glial cells, and neurons in the prefrontal cortex of patients suffering depression (Drevets et al, 1997; Ongur et al, 1998; Rajkowska et al, 1999). Neurons in the rodent mPFC undergo changes in their structure under the influence of stress or corticosterone (Radley et al, 2004; Wellman, 2001), psychoactive drugs (Robinson and Kolb, 1999), gonadal hormones (Forgie and Kolb, 2003), or changes in blood pressure (Vega et al, 2004). This neuronal remodeling in the MPFC may be mediated by changes in the expression of the polysialylated form of the neural cell adhesion molecule (PSA-NCAM), as it appears to occur in regions such as the hippocampus or the amygdala (Cordero et al, 2005; Pham et al, 2003). NCAM can incorporate long chains of PSA, which confer it antiadhesive properties (Rutishauser, 1996). Consequently, the expression of PSA-NCAM allows neurons to participate in plastic events such as neurite outgrowth (Zhang et al, 1992) or synaptic reorganization (Seki and Rutishauser, 1998). Recently, our laboratory has shown the presence of PSANCAM in certain neurons and the neuropil of the adult rat mPFC (Varea et al, 2005), suggesting that PSA-NCAM may also mediate the structural changes occurring in this region. 
Pyramidal neurons in the mPFC undergo dendritic remodeling after specific prefrontocortical serotonin (5-HT) depletion (Perez-Vega et al, 2000), indicating that neuronal structural plasticity in this cortical region may be mediated by serotoninergic transmission. General 5-HT depletion also reduces PSA-NCAM expression in the hippocampus, as well as in hypothalamic nuclei and basal ganglia (Brezun and Daszuta, 1999; Brezun and Daszuta, 2000), which indicates a relationship between 5-HT and PSA-NCAM expression. The mPFC receives a dense serotoninergic innervation (Azmitia and Segal, 1978; Steinbusch, 1981) and contains several 5-HT receptor subtypes (Pazos et al, 1985; Pazos and Palacios, 1985; Pompeiano et al, 1992). Consequently, this cortical region is a good target for antidepressants enhancing extracellular 5-HT levels, such as fluoxetine (Wong et al, 1974). Interestingly, a recent report has shown that treatment with fluoxetine, induces a robust increase in hippocampal pyramidal spine synapse density (Hajszan et al, 2005), indicating that this antidepressant may induce structural reorganization of neurons.

In order to better understand how neuronal structural plasticity in the MPFC is affected by depression and how this plasticity can be modulated by 5 -HT pharmacological manipulation, we have investigated the structural relationship between 5-HT fibers and PSA-NCAM immunoreactive neurons, the presence of 5-HT receptors in PSA-NCAM expressing neurons of the MPFC and the effects of chronic fluoxetine treatment in the expression of PSA-NCAM in the mPFC.

\section{MATERIALS AND METHODS}

\section{Animal Treatments and Histology}

Twenty-six male Sprague-Dawley rats (4 months old, $320 \pm 50 \mathrm{~g}$ Harlan Iberica) were used in this experiment. Animals were separated in the following groups: (i) six rats were used to study PSA-NCAM expression and its colocalization with 5-HT receptors using immunohistochemistry; (ii) 20 rats were used for fluoxetine and ondansetron treatment. All animal experimentation was conducted in accordance with the European Communities Council Directive of 24 November 1986 (86/609/EEC).

Rats were perfused transcardially under deep chloral hydrate anesthesia, with saline and then $4 \%$ paraformaldehyde in sodium phosphate buffer $0.1 \mathrm{M}, \mathrm{pH} 7.4$ (PB). After perfusion, the brains were extracted and cryoprotected with $30 \%$ sucrose in $\mathrm{PB}$. Coronal sections $(50 \mu \mathrm{m})$ were obtained with a sliding microtome and stored at $-20^{\circ} \mathrm{C}$ in $30 \%$ glycerol; 30\% ethylene glycol, 40\% PB until used.

\section{PSA-NCAM Immunohistochemistry}

Tissue was processed 'free-floating' for immunohistochemistry as follows. Briefly, sections were incubated for $1 \mathrm{~min}$ in an antigen unmasking solution ( $0.01 \mathrm{M}$ citrate buffer, $\mathrm{pH} 6)$ at $100^{\circ} \mathrm{C}$. After cooling down the sections to room temperature, they were incubated with $10 \%$ methanol, $3 \%$ $\mathrm{H}_{2} \mathrm{O}_{2}$ in phosphate buffered saline (PBS) for 10 min to block endogenous peroxidase activity. After this, sections were treated for $1 \mathrm{~h}$ with $5 \%$ normal donkey serum (NDS) (Jackson Laboratories) in PBS with $0.2 \%$ Triton-X100
(Sigma) and were incubated overnight at room temperature in mouse monoclonal IgM Men-B anti-PSA-NCAM antibody (1:1400; generous gift of $\mathrm{Dr} G$ Rougon). After washing, sections were incubated for $30 \mathrm{~min}$ with donkey anti-mouse IgM biotinilated antibody (Jackson Laboratories, 1:250), followed by an avidin-biotin-peroxidase complex (ABC, Vector Laboratories) for $30 \mathrm{~min}$ in PBS. Color development was achieved by incubating with $3,3^{\prime}$ diaminobenzidine tetrahydrochloride (DAB, Sigma) for $4 \mathrm{~min}$. PBS containing $0.2 \%$ Triton-X100 and 3\% NDS was used for primary and secondary antibodies dilution.

Pretreatment of the PSA-NCAM antibody with $\alpha-2,8$ linked sialic polymer (colominic acid, Sigma) overnight, or the primary antibody omission during the immunohistochemistry prevented all the labeling in the mPFC.

All of the sections studied passed through all procedures simultaneously, to minimize any differences from immunohistochemical staining itself.

\section{Double Immunofluorescence}

In order to characterize the spatial relationship between 5-HT immunoreactive fibers and PSA-NCAM immunoreactive neurons, and the expression of 5-HT receptors (5-HT1A, 5-HT2A, and 5-HT3) in PSA-NCAM immunoreactive neurons and neuropil in the medial prefrontal cortex, we have performed double immunohistochemistry using an anti-PSA-NCAM antibody and antibodies against 5-HT and its receptors. In general, sections were processed as described above, but the endogenous peroxidase block was omitted. The sections were incubated overnight with mouse monoclonal IgM anti-PSA-NCAM antibody (Men-B, $1.1400)$ and one of the following primary IgG antibodies: Rabbit anti-5-HT (1:1000, Affinity), rabbit anti-5-HT1A (1:100, Santa Cruz Biotechnology H-119), mouse anti5-HT2A (1:1000, Pharmigen), or rabbit anti-5-HT3 (1:250, Calbiochem). After washing, sections were incubated with donkey anti-mouse IgM, donkey anti-mouse IgG or donkey anti-rabbit IgG secondary antibodies conjugated with Alexa 488 or Alexa 555 (Molecular Probes, 1:200) in PBS containing $0.2 \%$ Triton $\mathrm{X}-100$ and $3 \%$ NDS. In the case of anti-5-HT2A the incubation with the two primary antibodies was made sequentially, first with the antibody against the receptor, washed and incubated with donkey anti-mouse IgG and then anti PSA-NCAM in order to avoid interactions between these two markers. The companies of origin have previously tested the specificity of the 5-HT and 5HT receptor antisera. Moreover, their specificity in rat tissue has been confirmed in different studies (Aznar et al, 2003; Morales et al, 1996; Morales and Bloom, 1997; Willins et al, 1997). Overnight incubation of 5-HT3 antibody with an excess of its immunogenic peptide resulted in a total absence of 5HT-3 immunostaining in the mPFC. Additional controls for the immunohistochemical procedure were carried out in our laboratory by omitting the primary or secondary antibodies in each step of the immunohistochemical protocol.

\section{Observation and Quantification of Double-Labeled Cells}

All sections processed for fluorescent immunohistochemistry were mounted on slides and coverslipped 
using Permafluor mounting medium (Immunon/Shandon, Pittsburgh, PA). Then, the sections were observed under a confocal microscope (Leica TCS-SP2). Z-series of optical sections $(1 \mu \mathrm{m}$ apart) were obtained using sequential scanning mode. These stacks were processed with LSM 5 Image Browser software. A 1-in-10 series of telencephalic sections from each animal were double-labeled as described. PSA-NCAM immunoreactive cells were first identified using conventional fluorescence microscopy. Then, a stack of confocal images covering all its three-dimensional extension was taken to confirm that PSA-NCAM labeling corresponded to cell somata. Fifty PSA-NCAM immunoreactive cells were analyzed in each case to determine the coexpression of PSA-NCAM and 5-HT receptors.

\section{Pharmacological Treatments}

Rats were divided into four groups $(n=5)$ and were treated (once daily) for 14 consecutive days, as follows. The first group was administered fluoxetine $(10 \mathrm{mg} / \mathrm{kg}$, intraperitoneal (i.p.), Sigma) and saline (30 min later). The second group received fluoxetine $(10 \mathrm{mg} / \mathrm{kg}, \mathrm{i} . \mathrm{p}$.$) and the 5-\mathrm{HT} 3$ receptor antagonist ondansetron $(2 \mathrm{mg} / \mathrm{kg}$, i.p., generous gift of Glaxo-Smithkline), $30 \mathrm{~min}$ later. The third group received an injection of saline and then ondansetron $(2 \mathrm{mg} /$ $\mathrm{kg}$ ), $30 \mathrm{~min}$ later. The fourth group received two injections of saline separated by $30 \mathrm{~min}$. The volume injected in every i.p. injection was $500 \mu \mathrm{l}$. All drugs used were dissolved in saline. After treatment, rats were killed and their brains were processed as described above.

\section{Quantification of PSA-NCAM Immunoreactive Neurons and Neuropil Immunoreactivity}

The number of PSA-NCAM expressing neurons in the different layers of the subdivisions of rat $\mathrm{mPFC}$ was estimated using a modified version of the fractionator method (West, 1993), as described before (Nacher et al, 2002a). We counted cells covering $100 \%$ of the sample area, that is, within each section, all labeled cells in the different subdivision and layers of the prefrontal cortex were counted. The fractionator sampling scheme refers to the methodology of examining one out of every 10 brain sections. Thus, our modification of the optical dissector combined with a 1:10 fractionator sampling is truly a modification of the optical fractionator method. 1:10 systematic-random series of sections covering the whole rostral to caudal extension of this structure were viewed on an Olympus CX41 microscope. Cell somata were identified and counted with a $\times 40$ objective. Cells appearing in the upper focal plane were omitted to prevent counting cell caps. The volume of the different areas analyzed was determined for each animal using the Cavalieri's principle (Gundersen and Jensen, 1987). Means were determined for each experimental group and the data were subjected to one-way ANOVAs followed by StudentNewman-Keuls post hoc tests.

In order to determine PSA-NCAM immunoreactivity intensity in the neuropil of medial prefrontal cortex, sections were examined under bright-field illumination, homogeneously lighted and digitalized using a CCD camera. Photographs were taken at $\times 20$ magnification. Gray levels were converted to optical densities (OD) using Image J software (NIH). Means were determined for each experimental group and the data were subjected to one-way ANOVAs followed by Student-Newman-Keuls post hoc tests.

\section{RESULTS}

\section{Body Weight}

Body weight changes were monitored at the beginning $(299.6 \pm 3.4 \mathrm{~g})$ and the end of the treatments (control: $356.0 \pm 8.6 \mathrm{~g}$; fluoxetine: $313.6 \pm 4.9 \mathrm{~g}$; fluoxetine + ondansetron: $325.0 \pm 6.4 \mathrm{~g}$; ondansetron: $357.2 \pm 7.0 \mathrm{~g}$ ). Fluoxetine treatment reduces significantly body weight gain and this reduction is prevented when ondansetron is coadministered with fluoxetine; ondansetron treatment alone did not interfere with body weight gain (One-way ANOVAs followed by Student-Newman-Keuls post hoc tests: $\left.\mathrm{F}_{4,20}=694.154, p<0.0001\right)$.

\section{Relationship between PSA-NCAM Immunoreactive Neurons and 5-HT Containing Fibers}

The distribution of PSA-NCAM immunoreactivity in the mPFC coincides with that previously described. The immunoreactivity was located in neurons, mainly characterized as interneurons, and in the neuropil with a clear lamination in the staining (Varea et al, 2005). PSA-NCAM immunoreactive neurons were located in all the layers of the mPFC but they were most abundant in deep layers. In general, PSA-NCAM immunoreactivity in the MPFC neuropil was moderately intense in layer I, weaker in layers II and III and intense in layers V-VI.

We have analyzed whether 5-HT immunoreactive fibers were closely apposed to PSA-NCAM immunoreactive neurons in the rat $\mathrm{mPFC}$ using double immunostaining with these two markers. Fibers containing 5-HT tended to be more dense in layers I-III and they were sparse in the inner layers. Some PSA-NCAM immunoreactive neurons presented apposed 5-HT fibers (Figure 1a). However, most commonly, 5-HT immunoreactive fibers did not appear to contact PSA-NCAM immunoreactive neurons (Figure 1b).

\section{5-HT Receptor Expression in PSA-NCAM Immunoreactive Neurons}

We analyzed the presence of 5-HT1A, 5-HT2A, and 5-HT3 receptors in PSA-NCAM immunoreactive neurons in the prefrontal cortex. These receptors were chosen because they are expressed by mPFC interneurons (Puig et al, 2004) and previous results from our laboratory have demonstrated that most PSA-NCAM immunoreactive cells in the rat MPFC are interneurons (Varea et al, 2005). 5-HT1A and 5-HT2A receptors are also expressed by pyramidal neurons, while 5-HT3 receptor is only expressed by interneurons (Puig et al, 2004).

None of the PSA-NCAM immunoreactive neurons analyzed expressed 5-HT1A (Figure 1c) or 5-HT2A receptors (Figure 1d). 5-HT2A was widely expressed by pyramidal neurons in this cerebral region (Figure 1e). By contrast, 94 $\%$ of the PSA-NCAM immunoreactive neurons also expressed the 5-HT3 receptor (Figures if and g). 

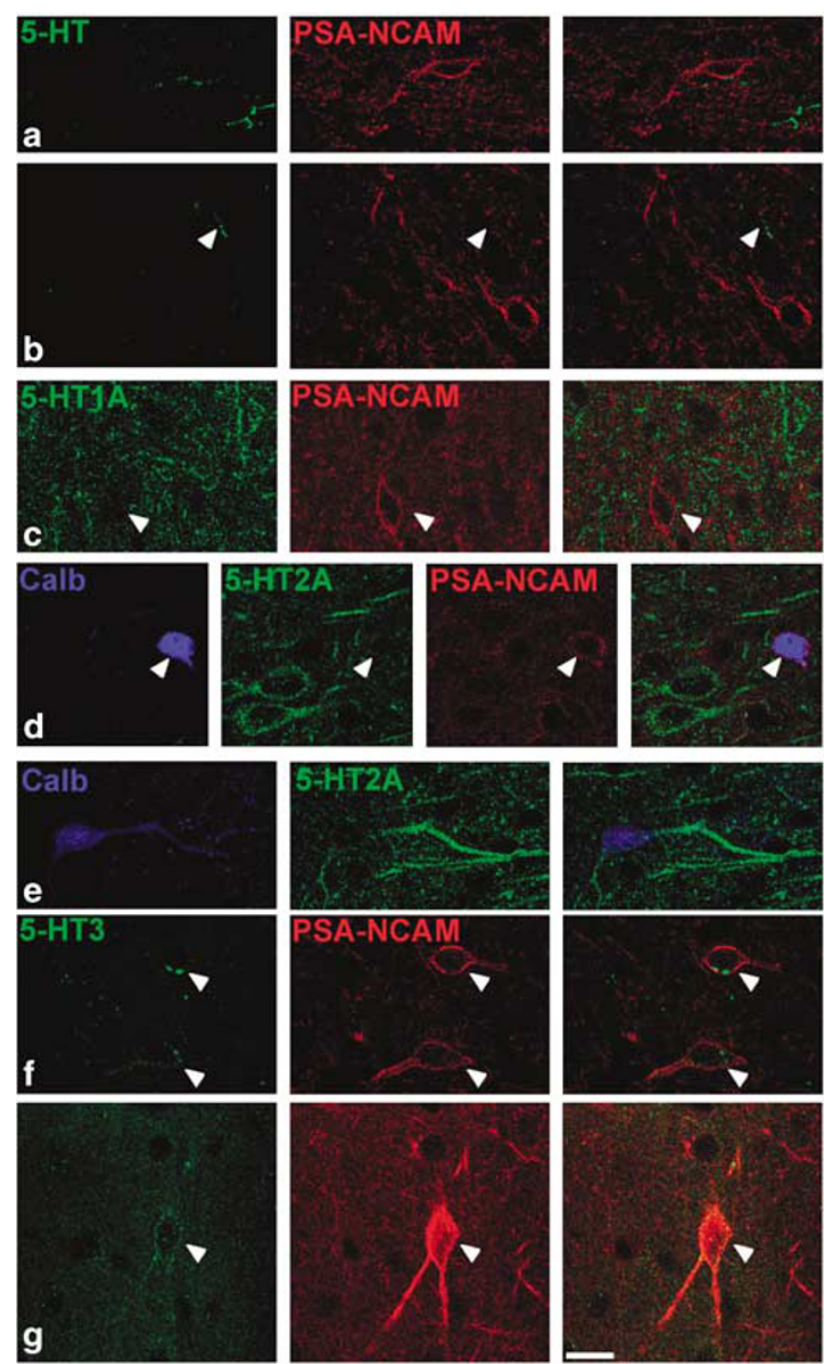

Figure I Confocal microscopic analysis of PSA-NCAM immunoreactive neurons in the mPFC. (a) PSA-NCAM immunoreactive neuron in the prelimbic cortex layer VI. Observe the presence of 5-HT fibers apposed to the neuron. (b) PSA-NCAM immunoreactive neuron in the cingulate cortex layer III. Note that 5-HT immunoreactive fibers (arrowhead) are not in the close vicinity of the neuronal soma. (c) Cingulate cortex layer VI. Double PSA-NCAM/5-HTIA immunohistochemistry. Observe the lack of colocalization between these two markers. Arrowhead indicates the presence of a PSA-NCAM immunoreactive neuronal soma. (d) Triple immunohistochemistry PSA-NCAM/5-HT2A/calbindin D28k in the cingulate cortex layer III. A PSA-NCAM immunoreactive neuron expresses calbindin but lacks 5-HT2A expression (arrowhead). Note the presence of two 5-HT2A immunoreactive cells on the bottom left side of the picture, which lack PSA-NCAM and calbindin D-28k immunoreactivity. (e) Calbindin D-28k/5-HT2A immunoreactive neuron in the cingulate cortex layer III. (f) PSA-NCAM/5-HT3 immunoreactive neuron in the prelimbic cortex layer $\vee$ (arrowhead indicates the presence of two PSA-NCAM immunoreactive neurons displaying 5-HT3 immunoreactivity). (g) PSANCAM/5-HT3 immunoreactive neuron in the cingulate cortex layer III (arrowhead indicates the presence of 5-HT3 immunoreactive neurons displaying PSA-NCAM immunoreactivity). Scale bar: $10 \mu \mathrm{m}$. All photographs in this figure correspond to single optical sections taken from Z-stacks.

\section{Pharmacological Treatments}

In order to check whether changes in extracellular levels of 5 -HT could affect the expression of PSA-NCAM in the
mPFC, rats were chronically treated with fluoxetine for 14 days and PSA-NCAM immunoreactive neurons as well as neuropil staining were analyzed. As most PSA-NCAM immunoreactive neurons in the MPFC expressed 5-HT3 receptors we have also evaluated the effects of ondansetron, a 5-HT3 receptor antagonist, on PSA-NCAM expression, when used alone or in combination with fluoxetine.

Chronic fluoxetine treatment induced a general tendency towards an increase in the number of PSA-NCAM immunoreactive neurons in the mPFC (Figure 2a, $\left.\mathrm{F}_{3,21}=5.36, p<0.067\right)$. Separated analysis of the different layers in the whole $\mathrm{mPFC}$ revealed that this increase in the number of immunoreactive neurons is only statistically significant in layers II-III. Coadministration of fluoxetine and ondansetron or ondansetron alone did not induce significant changes the number of PSA-NCAM expressing neurons in the whole MPFC; however, the numbers of PSANCAM immunoreactive neurons found in every layer after these treatments were significantly lower than that of fluoxetine-treated rats (Figure 2a, layer $\mathrm{I}: \mathrm{F}_{3,21}=0.57$, $p<0.6$; layers II-III: $\mathrm{F}_{3,21}=6.32, p<0.002$; layers V-VI: $\left.\mathrm{F}_{3,21}=4.34, p<0.015\right)$.

The individual analysis of the subdivisions of the mPFC revealed that the infralimbic cortex showed a statistically significant increase in the number of PSA-NCAM immunoreactive cells in whole infralimbic cortex and in layers II-III and a tendency towards an increase in the rest of the layers. Combined treatment with fluoxetine and ondansetron did not change the number of PSA-NCAM immunoreactive neurons in any of the layers analyzed. Similar results were obtained with ondansetron treatment alone, except in layers II-III, where a tendency towards a decrease in the number of labeled cells was observed. Animals treated with both ondansetron and fluoxetine (whole infralimbic cortex and layers II-III) or with ondansetron alone (whole infralimbic cortex and layers II-III and V-VI) exhibited significantly less PSA-NCAM immunoreactive neurons than those treated only with fluoxetine (Figure $2 \mathrm{~b}$, layer I: $\mathrm{F}_{3,24}=0.7$, $p<0.55$; layers II-III: $\mathrm{F}_{3,24}=6.18, p<0.0029$; layers $\mathrm{V}$-VI: $\left.\mathrm{F}_{3,21}=3.37, p<0.024\right)$.

In the prelimbic cortex (Figure 2c), no statistically significant differences in the number of PSA-NCAM expressing neurons were found between each of the treatments studied and the controls. However, the prelimbic cortex as a whole and layers II-III and V-VI showed a slight, but not statistically significant, increase in the number of PSA-NCAM immunoreactive neurons after chronic fluoxetine treatment. Animals treated with both ondansetron and fluoxetine exhibited significantly less PSA-NCAM immunoreactive neurons than those treated only with fluoxetine in layers V-VI (Figure 2c, layer I: $\mathrm{F}_{3,24}=0.75, p<0.52$; layers II-III: $\mathrm{F}_{3,24}=1.64, p<0.20$; layers V-VI: $F_{3,21}=3.07, p<0.047$ ).

Similar results were obtained in the cingulate cortex (Figure 2d). There was a slight, but not statistically significant, increase in the number of PSA-NCAM immunoreactive neurons after chronic fluoxetine treatment in the prelimbic cortex as a whole, and in layers II-III and V-VI. The rest of the treatments did not induce changes in the number of PSA-NCAM immunoreactive neurons in the cingulate cortex. However, fluoxetine-treated animals had significantly more PSA-NCAM immunoreactive neurons in 
a

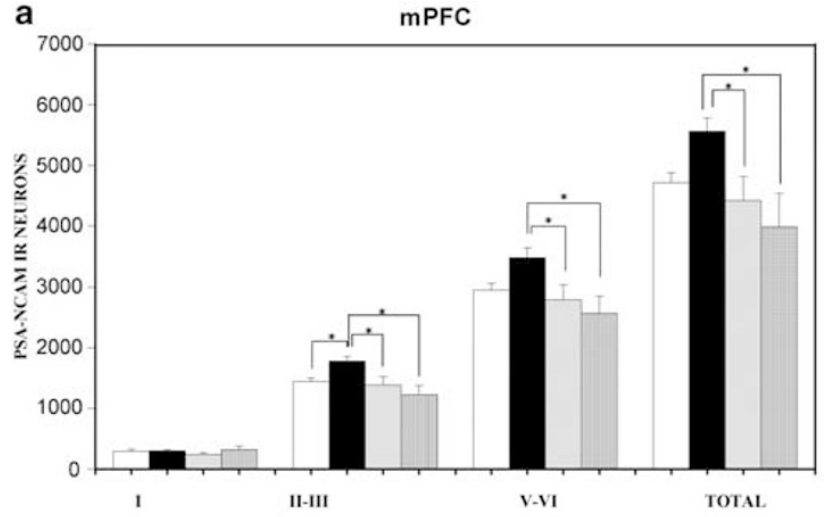

C

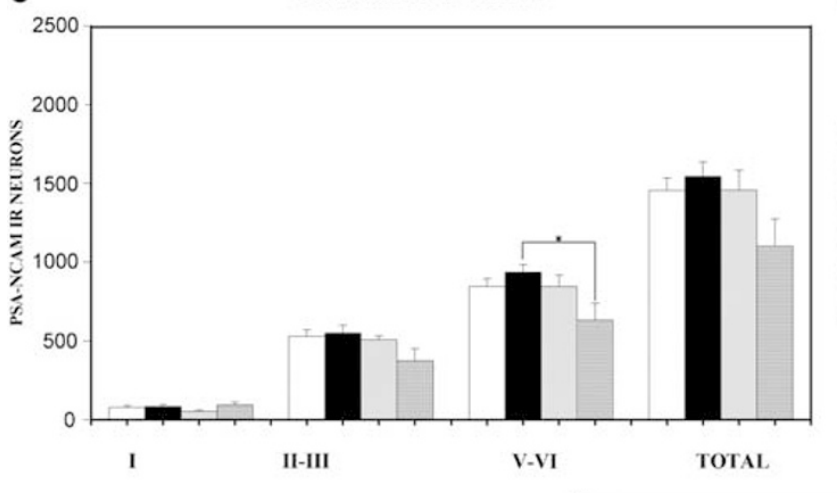

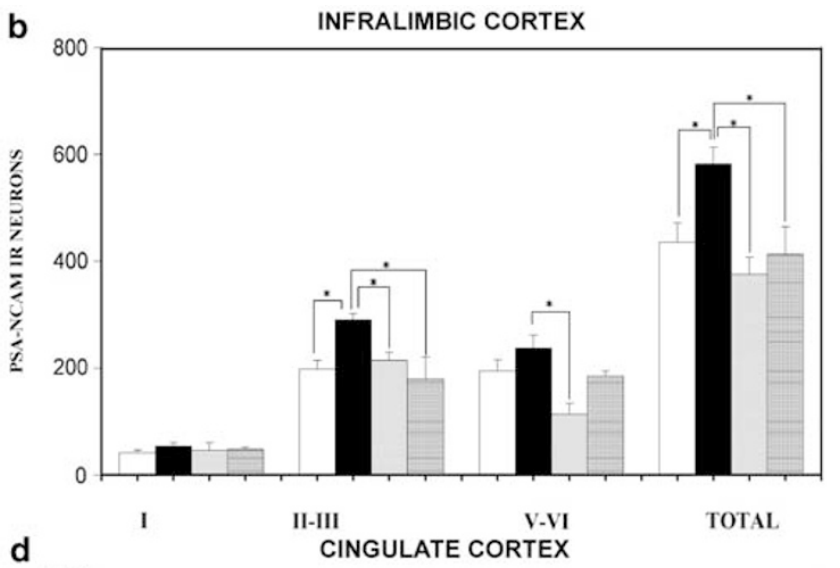

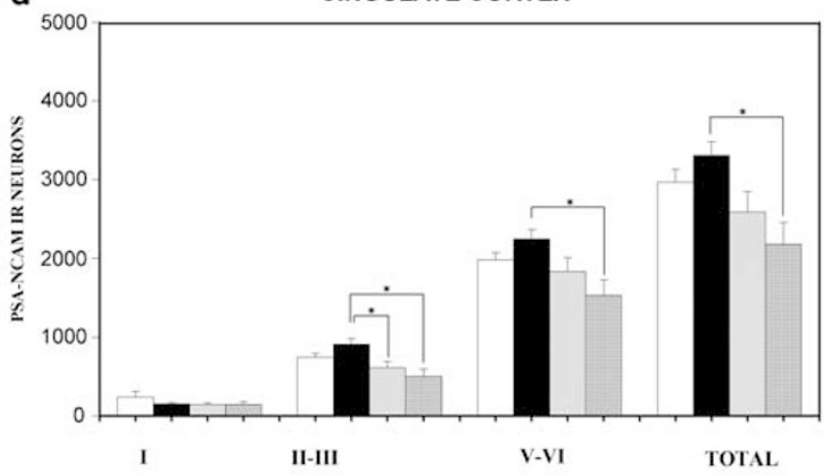

Figure 2 Graphs showing the changes in the number of PSA-NCAM immunoreactive neurons in medial prefrontal cortex: (a) Whole medial prefrontal cortex, (b) Infralimbic cortex, (c) Prelimbic cortex, (d) cingulate cortex. *Statistically significant, one-way ANOVA followed by Student-Newman-Keuls post hoc tests.

layers II-III than those treated with both fluoxetine and ondansetron. Animals treated with both ondansetron and fluoxetine exhibited significantly less PSA-NCAM immunoreactive neurons in the whole cingulate cortex and in layers II-III and V-VI than those treated only with fluoxetine (layer I: $\mathrm{F}_{3,24}=0.75, p<0.52$; layer II-III: $\mathrm{F}_{3,24}=1.64$, $p<0.20$; layer V-VI: $\left.F_{3,21}=3.07, p<0.047\right)$.

We also checked whether chronic treatment with fluoxetine affected the intensity of PSA-NCAM immunoreactivity in the neuropil. As a general feature, there was an increase in PSA-NCAM immunoreactivity in all the layers of all the subdivisions of the rat $\mathrm{mPFC}$ as a consequence of fluoxetine treatment, which was not observed when ondansetron was coadministered with fluoxetine (Figure 3).

In the infralimbic cortex the increase in PSA-NCAM immunoreactivity after fluoxetine treatment was statistically significant in every layer studied and it was prevented when ondansetron was coadministered with fluoxetine; ondansetron treatment alone did not change significantly the expression of PSA-NCAM in the infralimbic cortex, although there was a general tendency towards a decrease. Animals treated with fluoxetine-ondansetron or ondansetron alone showed significantly reduced immunoreactivity (in every layer studied) when compared with those treated only with fluoxetine (Figure 3a, layer I: $\mathrm{F}_{3,19}=6.31$, $p<0.0037$; layer II: $\mathrm{F}_{3,19}=5.94, p<0.0049$; layer III: $\mathrm{F}_{3,19}=8.57, p<0.0008$; layer $\mathrm{V}: \mathrm{F}_{3,19}=6.47, p<0.0033$; layer VI: $\left.F_{3,19}=6.59, p<0.0031\right)$.
In the prelimbic cortex fluoxetine also induced a statistically significant increase in PSA-NCAM immunoreactivity in every layer studied, which was prevented when ondansetron was coadministered with fluoxetine; ondansetron treatment alone did not change significantly the expression of PSA-NCAM in any layer of the prelimbic cortex, although there was a general tendency towards a decrease. Animals treated with fluoxetine-ondansetron or ondansetron alone showed significantly reduced immunoreactivity (in every layer studied) when compared with those treated only with fluoxetine (Figure $3 \mathrm{~b}$, layer I: $\mathrm{F}_{3,19}=10.25, p<0.0003$; layer II: $\mathrm{F}_{3,19}=9.25, p<0.0006$; layer III: $F_{3,19}=12.65, p<0.0001$; layer $\mathrm{V}: \mathrm{F}_{3,19}=12.09$, $p<0.0001$; layer VI: $\left.\mathrm{F}_{3,19}=9.84, p<0.0004\right)$.

Finally, in the neuropil of both the ventral and the dorsal cingulate cortices we have observed a statistically significant increase in PSA-NCAM expression in every layer after chronic fluoxetine treatment. These increases were not present when ondansetron was coadministered with fluoxetine. Interestingly, chronic ondansetron administration alone decreased significantly PSA-NCAM expression in layers II and III of the ventral cingulate cortex and there was also a tendency towards a decrease in the rest of the layers of both cingulate cortices. Animals treated with fluoxetineondansetron or ondansetron alone showed significantly reduced immunoreactivity (in every layer studied) when compared with those treated only with fluoxetine (ventral cingulate cortex: layer I: $\mathrm{F}_{3,19}=4.86, p<0.011$; layer II: 

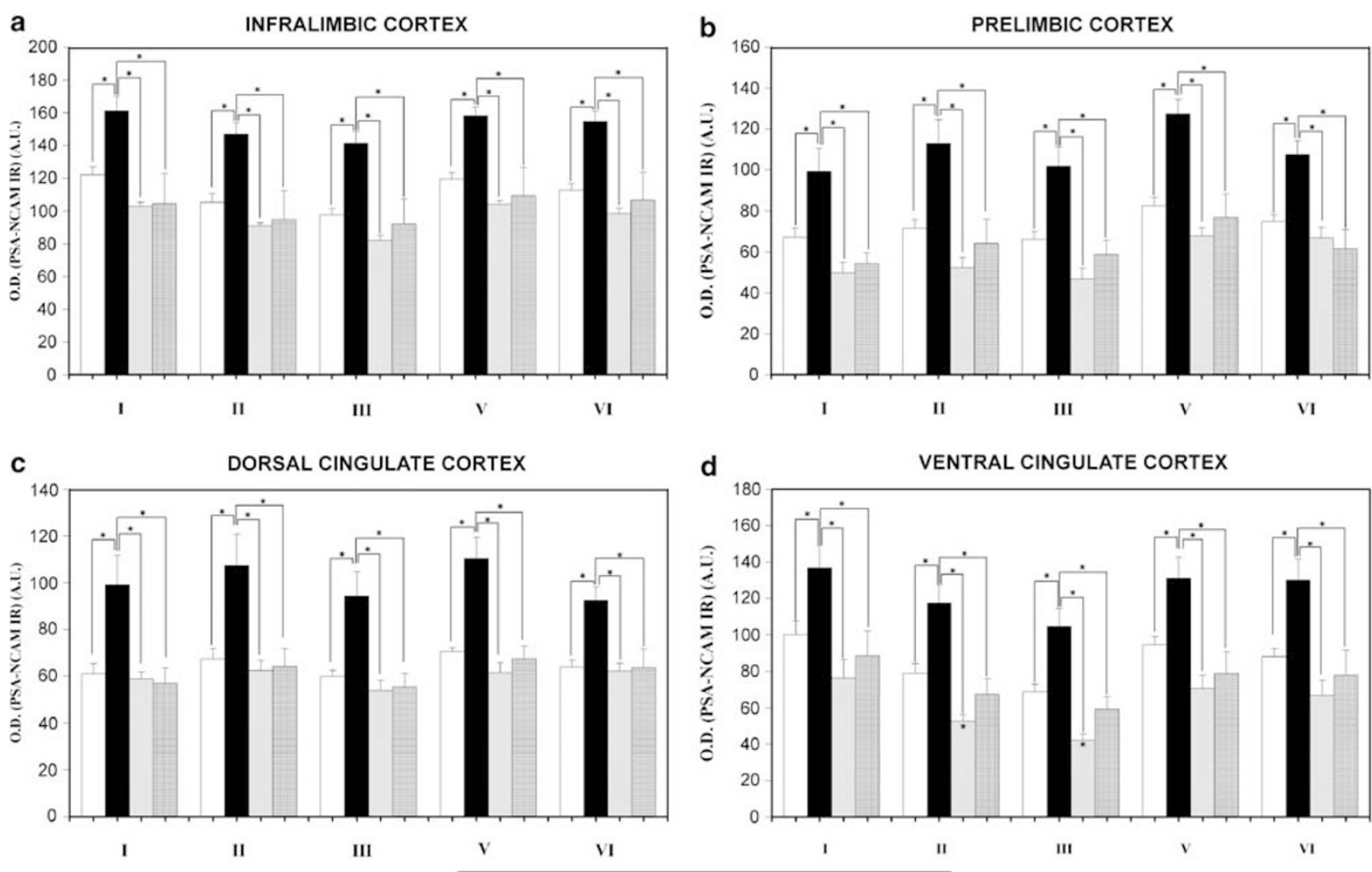

d

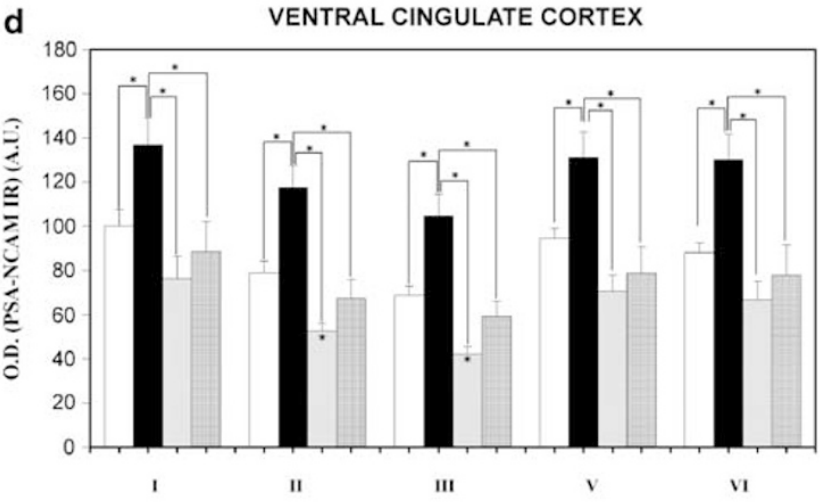

Figure 3 Graphs representing the changes in the intensity of PSA-NCAM neuropil immunostaining in the mPFC. (a) Infralimbic cortex, (b) prelimbic cortex, (c) dorsal cingulate cortex. (d) Ventral cingulate cortex (asterisks in ondansetron bars indicate significant differences from control group). *Statistically significant, one-way ANOVA followed by Student-Newman-Keuls post hoc tests.

$\mathrm{F}_{3,19}=12.70, p<0.0001$; layer III: $\mathrm{F}_{3,19}=16.53, p<0.0001$; layer V: $\mathrm{F}_{3,19}=8.37, p<0.0009$; layer $\mathrm{VI}: \mathrm{F}_{3,19}=7.58$, $p<0.0016$. dorsal cingulate cortex: layer $\mathrm{I}: \mathrm{F}_{3,19}=4.14$, $p<0.02$; layer II: $\mathrm{F}_{3,19}=4.25, p<0.01$; layer III: $\mathrm{F}_{3,19}=7.44$, $p<0.0017$; layer $\mathrm{V}$ : $\mathrm{F}_{3,19}=15.63, p<0.0001$; layer $\mathrm{VI}$ : $\left.\mathrm{F}_{3,19}=6.45, p<0.003\right)$.

\section{DISCUSSION}

This report describes a clear effect of chronic fluoxetine treatment on the expression of PSA-NCAM in the adult rat mPFC. We also provide evidence that PSA-NCAM immunoreactive neurons in this cortical region express 5-HT3 receptors and that the effects of fluoxetine treatment on PSA-NCAM expression can be prevented by coadministration of a 5-HT3 antagonist. The mechanisms by which 5-HT may modulate PSA-NCAM expression in the $\mathrm{mPFC}$ are discussed. We also analyze how these changes in PSANCAM expression may modulate neuronal structural plasticity. Finally, the putative functional consequences of this antidepressant action are discussed.

\section{Involvement of 5-HT in the Modulation of PSA-NCAM Expression in the mPFC}

Our results demonstrate that the expression of PSA-NCAM in the $\mathrm{mPFC}$ is modulated by chronic fluoxetine treatment.
As this compound acts inhibiting 5-HT reuptake and, consequently, increasing the extracelullar concentrations of 5-HT (Wong et al, 1974), it is very likely that enhanced 5 -HT binding to its receptors in certain mPFC neurons may induce the reported increase in PSA-NCAM expression. The adult rat $\mathrm{mPFC}$ is an important target of $5-\mathrm{HT}$ action, because it receives a dense serotoninergic innervation from the raphe nuclei (Azmitia and Segal, 1978; Steinbusch, 1981) and it contains several receptor subtypes for 5-HT (Pazos et al, 1985; Pazos and Palacios, 1985; Pompeiano et al, 1992). Moreover, the prefrontal cortex neurons project back to the brain nuclei that synthesize 5-HT, inducing its release (Jankowski and Sesack, 2004; Peyron et al, 1998; Takagishi and Chiba, 1991; Vertes, 2004). Previous reports have already demonstrated a link between PSA-NCAM expression and 5-HT: depletion of 5-HT reduces PSA-NCAM expression in the hippocampus, certain hypothalamic nuclei and the basal ganglia (Brezun and Daszuta, 1999; Brezun and Daszuta, 2000). Although the mechanism by which 5-HT modulates PSA-NCAM expression is not yet known, 5-HT may influence PSA-NCAM expression by acting directly on receptors located in certain neurons. Our results provide evidence that PSA-NCAM immunoreactive cells in the $\mathrm{mPFC}$ express 5-HT-3 receptors and thus it may be feasible that 5-HT could act directly through this type of receptor to upregulate PSA-NCAM expression. In fact, we have found that coadministration of fluoxetine and ondansetron (a 5-HT3 receptor antagonist) prevents the 
induction of PSA-NCAM expression induced by fluoxetine treatment. Moreover, chronic ondansetron administration alone is sufficient to induce a decrease in PSA-NCAM expression in certain layers of the cingulate cortex. Interestingly, recent results from our lab indicate that precisely these layers are the first to display changes in PSANCAM expression after chronic stress (Gomez-Climent and Nacher, unpublished results).

Fluoxetine appears to induce a general increase in PSANCAM expression, which is reflected by an increase in the number of positive neuronal somata and an increase in neuropil immunoreactivity. This may indicate an upregulation of PSA-NCAM expression in neuronal elements in which PSA-NCAM was not previously detected, because its level was under the detection limits of the technique employed, or to 'de novo' expression of PSA-NCAM in elements, which only expressed PSA-NCAM after fluoxetine treatment. We have not yet identified clearly the nature of PSA-NCAM expressing elements in the $\mathrm{mPFC}$ neuropil: they may correspond to neurites of intrinsic PSA-NCAM expressing neurons or they may correspond to processes coming from neurons located in areas that project to the mPFC.

We have found that 5-HT expressing fibers usually do not contact PSA-NCAM expressing neurons, suggesting that 5-HT action may not need direct contact between 5-HT expressing fibers and PSA-NCAM expressing cells. This result is in accordance with previous reports that indicate an extra-synaptic action of 5-HT, because this neurotransmitter is able to diffuse considerable distances from its point of release (De Miguel and Trueta, 2005) and consequently, once secreted, 5-HT can act on wide areas of the CNS. In fact, 5-HT receptors can be found widely distributed in the mPFC (Morales et al, 1996; Puig et al, 2004; Santana et al, 2004) and we detect increases in PSANCAM expression, specially in the neuropil, in every layer of the mPFC subdivisions. Thus, although the density of 5-HT fibers in superficial layers of the MPFC is higher, the increases in PSA-NCAM expression detected in deeper layers may also be explained by 5 -HT action.

A previous report has described that PSA-NCAM expressing neurons in the rat $\mathrm{mPFC}$ are mainly interneurons and that pyramidal neurons do not appear to express this molecule (Varea et al, 2005). Interneurons in the mPFC can be targets of 5-HT action because they are known to express 5-HT1A, 5-HT2A, and 5-HT3 receptors (Morales et al, 1996; Puig et al, 2004; Santana et al, 2004). Our present results indicate that $94 \%$ of PSA-NCAM expressing neurons in the mPFC express the 5-HT-3 receptor and that they lack 5HT1A and 5-HT2A receptors, suggesting that 5-HT actions on PSA-NCAM expression occur through 5-HT3 receptors. The 5-HT3 receptor is a ligand-gated ionic channel (Derkach et al, 1989), permeable to monovalent cations (Peters and Lambert, 1989) and excitatory to interneurons (Puig et al, 2004). Previous reports have also identified 5-HTR3 expression in cortical interneurons expressing CCK, calbindin, and calretinin, although it is not present in parvalbumin expressing interneurons (Morales and Bloom, 1997). These results are in accordance with our previous report describing calbindin immunoreactivity in most mPFC PSA-NCAM expressing neurons and the absence of parvalbumin immunostaining in these cells (Varea et al, 2005).
The mechanism of 5-HT action on PSA-NCAM expression may be, at least partially, explained through the participation of 5-HT3 receptors. The increase in the extracellular concentration of 5-HT induces depolarization of neurons through 5-HT3 receptors, increasing neuronal activity (Puig et al, 2004), which might lead to increased PSA-NCAM expression.

Alternatively, the actions of 5-HT on PSA-NCAM expression may also be mediated by BDNF. Activation of 5-HT receptors can induce the expression of BDNF (see Mattson et al (2004) for review) and the interaction between this neurotrophin and PSA-NCAM is believed to be critical in regulating neuronal plasticity (Kiss et al, 2001).

\section{Involvement of PSA-NCAM Expression in MPFC Neuronal Structural Plasticity}

The presence of PSA-NCAM in the membrane of certain neurons may allow them to undergo plastic changes such as dendritic or spine remodeling. NCAM has the ability to incorporate long chains of polysialic acid (NCAM) conferring it anti-adhesive properties (Rutishauser, 1996). PSANCAM regulates CNS structural plasticity, allowing neurons to participate in plastic events such as axonal growth (Zhang et al, 1992) or synaptic reorganization (Miragall et al, 1988; Seki and Rutishauser, 1998). In adult animals, this molecule is expressed in cerebral regions that are undergoing some kind of structural plasticity, such as the hypothalamo-neurohypophyseal system (Theodosis et al, 1994), the olfactory bulb (Miragall et al, 1988) the piriform and entorhinal cortices (Seki and Arai, 1991a), the amygdala (Nacher et al, 2002b), and the hippocampus (Seki and Arai, 1991b). Previous reports have demonstrated the existence of structural plasticity in neurons of the mPFC, namely their ability to reorganize their structure under stress conditions (Brown et al, 2005; Cook and Wellman, 2004; Radley et al, 2004), or after corticosterone administration (Wellman, 2001). Moreover, the morphology of these neurons is also sensitive to alterations in blood pressure (Vega et al, 2004). PSA-NCAM is expressed in certain neurons and the neuropil of the rat mPFC (Varea et al, 2005), suggesting that some of the structural plasticity occurring in this cortical region may be mediated by this molecule.

Interestingly, although the expression of PSA-NCAM seems to be exclusive of mPFC interneurons (Varea et al, 2005), all the structural changes reported in the MPFC were only described in pyramidal neurons (Radley et al, 2005). However, at least some of these principal neurons are surrounded by PSA-NCAM immunoreactive puncta that may correspond to synapses (Varea et al, 2005). It is thus feasible that the presence of PSA-NCAM in certain synapses contacting mPFC pyramidal neurons may promote a transitory detachment, leading to a reorganization of pyramidal neuron dendrites. Alternatively, the increase in PSA-NCAM expression may indicate the presence of a sprouting phenomenon preceding or induced by a putative increase in the dendritic branching or in the number of dendritic spines in pyramidal neurons. In fact, chronic treatment with fluoxetine induces a robust increase in dendritic spine synapse density in CA1 and CA3 hippocampal pyramidal neurons (Hajszan et al, 2005) and similar effects may occur in the mPFC. Moreover, prefrontocortical 
5-HT depletion, which may produce the opposite effects of fluoxetine, induces dendritic atrophy of mPFC pyramidal neurons (Perez-Vega et al, 2000). It is thus possible that 5-HT may act directly on pyramidal neurons, forcing them to modify its structure and that the increase in PSA-NCAM expression may be a response of interneurons to these structural changes.

Several studies have described opposite actions of chronic stress and antidepressant treatment on neuronal structural plasticity. One of the best studied regions is the hippocampus, in which chronic stress induces dendritic atrophy in pyramidal cells (McEwen, 2000). This atrophy is prevented by antidepressant treatment (Magarinos et al, 1999) and a recent report has described that fluoxetine treatment alone is capable of increasing spine synapses in these pyramidal neurons (Hajszan et al, 2005). As chronic stress also induces dendritic atrophy and spine loss in mPFC pyramidal neurons (Radley et al, 2004; Wellman, 2001), it is feasible that fluoxetine treatment may induce the opposite effect. The fact that chronic fluoxetine treatment induces an increase in synaptohysin (a protein specific of synapses) expression in the cerebral cortex (Rapp et al, 2004), gives support to this hypothesis. However, studies on the structure of mPFC neurons after antidepressant treatment should be performed to clarify this matter. Moreover, the identification of the phenotype and connectivity of the neurons that are expressing PSA-NCAM is a critical point that also deserves careful studies in the future. In any case, the observed changes in PSA-NCAM expression most likely reflect the reorganization of neuronal circuitry that is supposed to occur after antidepressive treatment (Castren, 2005).

\section{Functional Implications of Fluoxetine Treatment and Structural Plasticity}

Although the suggestion that mood disorders were caused by a neurochemical imbalance has been widely accepted over the last few decades, recent evidences have lead to the emergence of a new hypothesis, which suggests that dysfunction in neuronal plasticity may underlie the etiology of depression (Castren, 2005; Mattson et al, 2004). The basis for the neurochemical hypothesis of depression came from the finding that certain antidepressants act increasing the extracelullar concentrations of 5-HT and noradrenaline in the CNS (monoamine hypothesis). However, the relationship between depression and monoamine concentrations appeared to be more complex than previously thought. Although the effects of antidepressants on monoamine metabolism occur rapidly, the therapeutic effects of antidepressants usually need weeks to take place (Nestler, 1998). This delay and the recent finding that antidepressants induce neuronal structural plasticity add support to the neuroplastic hypothesis.

Although we are still far from understanding the cellular bases of depression, the presence of PSA-NCAM and its modulation by fluoxetine open a new perspective in the study of this mood disorder. Of particular interest is the recent development of PSA-NCAM mimotope peptides, which may be promising therapeutic drugs for the treatment of mood disorders (Cambon et al, 2004; Torregrossa et al, 2004). Future directions should include the study of PSA-NCAM expression in the brains of depressed patients and research directed to deepen into the molecular cascades leading to the enhanced PSA-NCAM expression found after antidepressant treatment.

\section{ACKNOWLEDGEMENTS}

We are grateful to Dr G Rougon for her kindly gift of antiPSA-NCAM antibody and to Mrs Sabina Wolsky for her help with the histological procedures. Ondansetron was generously supplied by Glaxo-Smithkline. This study was supported by the following grants: GV04A-134, GV04A-076, GVGrupos03/119, MEC BFI2003-01254, and MEC BFU200400931.

\section{REFERENCES}

Azmitia EC, Segal M (1978). An autoradiographic analysis of the differential ascending projections of the dorsal and median raphe nuclei in the rat. J Comp Neurol 179: 641-667.

Aznar S, Qian Z, Shah R, Rahbek B, Knudsen GM (2003). The 5HT1A serotonin receptor is located on calbindin- and parvalbumin-containing neurons in the rat brain. Brain Res 959: 58-67.

Bearden CE, Hoffman KM, Cannon TD (2001). The neuropsychology and neuroanatomy of bipolar affective disorder: a critical review. Bipolar Disord 3: 106-150.

Brezun JM, Daszuta A (1999). Serotonin depletion in the adult rat produces differential changes in highly polysialylated form of neural cell adhesion molecule and tenascin- $\mathrm{C}$ immunoreactivity. J Neurosci Res 55: 54-70.

Brezun JM, Daszuta A (2000). Serotonergic reinnervation reverses lesion-induced decreases in PSA-NCAM labeling and proliferation of hippocampal cells in adult rats. Hippocampus 10: 37-46.

Brown SM, Henning S, Wellman CL (2005). Mild, short-term stress alters dendritic morphology in rat medial prefrontal cortex. Cereb Cortex 11: 1714-1722.

Cambon K, Hansen SM, Venero C, Herrero AI, Skibo G, Berezin V et al (2004). A synthetic neural cell adhesion molecule mimetic peptide promotes synaptogenesis, enhances presynaptic function, and facilitates memory consolidation. J Neurosci 24: 4197-4204.

Castren E (2005). Is mood chemistry? Nat Rev Neurosci 6: 241-246.

Cook SC, Wellman CL (2004). Chronic stress alters dendritic morphology in rat medial prefrontal cortex. J Neurobiol 60: 236-248.

Cordero MI, Rodriguez JJ, Davies HA, Peddie CJ, Sandi C, Stewart MG (2005). Chronic restraint stress down-regulates amygdaloid expression of polysialylated neural cell adhesion molecule. Neuroscience 133: 903-910.

De Miguel FF, Trueta C (2005). Synaptic and extrasynaptic secretion of serotonin. Cell Mol Neurobiol 25: 297-312.

Derkach V, Surprenant A, North RA (1989). 5-HT3 receptors are membrane ion channels. Nature 339: 706-709.

Drevets WC, Price JL, Simpson Jr JR, Todd RD, Reich T, Vannier $M$ et al (1997). Subgenual prefrontal cortex abnormalities in mood disorders. Nature 386: 824-827.

Duman RS, Malberg J, Thome J (1999). Neural plasticity to stress and antidepressant treatment. Biol Psychiatry 46: 1181-1191.

Fava M, Kendler KS (2000). Major depressive disorder. Neuron 28: 335-341.

Forgie ML, Kolb B (2003). Manipulation of gonadal hormones in neonatal rats alters the morphological response of cortical neurons to brain injury in adulthood. Behav Neurosci 117: 257-262.

Gundersen HJ, Jensen EB (1987). The efficiency of systematic sampling in stereology and its prediction. J Microsc 147(Part 3) 229-263. 
Hajszan T, MacLusky NJ, Leranth C (2005). Short-term treatment with the antidepressant fluoxetine triggers pyramidal dendritic spine synapse formation in rat hippocampus. Eur J Neurosci 21: 1299-1303.

Jankowski MP, Sesack SR (2004). Prefrontal cortical projections to the rat dorsal raphe nucleus: ultrastructural features and associations with serotonin and gamma-aminobutyric acid neurons. J Comp Neurol 468: 518-529.

Kiss JZ, Troncoso E, Djebbara Z, Vutskits L, Muller D (2001). The role of neural cell adhesion molecules in plasticity and repair. Brain Res Brain Res Rev 36: 175-184.

Magarinos AM, Deslandes A, McEwen BS (1999). Effects of antidepressants and benzodiazepine treatments on the dendritic structure of CA3 pyramidal neurons after chronic stress. Eur J Pharmacol 371: 113-122.

Manji HK, Duman RS (2001). Impairments of neuroplasticity and cellular resilience in severe mood disorders: implications for the development of novel therapeutics. Psychopharmacol Bull 35: 5-49.

Manji HK, Drevets WC, Charney DS (2001). The cellular neurobiology of depression. Nat Med 7: 541-547.

Manji HK, Moore GJ, Rajkowska G, Chen G (2000). Neuroplasticity and cellular resilience in mood disorders. Mol Psychiatry 5: 578-593.

Mattson MP, Maudsley S, Martin B (2004). BDNF and 5-HT: a dynamic duo in age-related neuronal plasticity and neurodegenerative disorders. Trends Neurosci 27: 589-594.

McEwen BS (2000). The neurobiology of stress: from serendipity to clinical relevance. Brain Res 886: 172-189.

Miragall F, Kadmon G, Husmann M, Schachner M (1988). Expression of cell adhesion molecules in the olfactory system of the adult mouse: presence of the embryonic form of N-CAM. Dev Biol 129: 516-531.

Morales M, Bloom FE (1997). The 5-HT3 receptor is present in different subpopulations of GABAergic neurons in the rat telencephalon. J Neurosci 17: 3157-3167.

Morales M, Battenberg E, de Lecea L, Bloom FE (1996). The type 3 serotonin receptor is expressed in a subpopulation of GABAergic neurons in the rat neocortex and hippocampus. Brain Res 731: 199-202.

Muller D, Nikonenko I, Jourdain P, Alberi S (2002). LTP, memory and structural plasticity. Curr Mol Med 2: 605-611.

Nacher J, Alonso-Llosa G, Rosell D, McEwen B (2002a). PSANCAM expression in the piriform cortex of the adult rat. Modulation by NMDA receptor antagonist administration. Brain Res 927: 111-121.

Nacher J, Lanuza E, McEwen BS (2002b). Distribution of PSANCAM expression in the amygdala of the adult rat. Neuroscience 113: $479-484$.

Nestler EJ (1998). Antidepressant treatments in the 21st century. Biol Psychiatry 44: 526-533.

Ongur D, Drevets WC, Price JL (1998). Glial reduction in the subgenual prefrontal cortex in mood disorders. Proc Natl Acad Sci USA 95: 13290-13295.

Pazos A, Palacios JM (1985). Quantitative autoradiographic mapping of serotonin receptors in the rat brain. I. Serotonin-1 receptors. Brain Res 346: 205-230.

Pazos A, Cortes R, Palacios JM (1985). Quantitative autoradiographic mapping of serotonin receptors in the rat brain. II. Serotonin-2 receptors. Brain Res 346: 231-249.

Perez-Vega MI, Feria-Velasco A, Gonzalez-Burgos I (2000). Prefrontocortical serotonin depletion results in plastic changes of prefrontocortical pyramidal neurons, underlying a greater efficiency of short-term memory. Brain Res Bull 53: 291-300.

Peters JA, Lambert JJ (1989). Electrophysiology of 5-HT3 receptors in neuronal cell lines. Trends Pharmacol Sci 10: 172-175.

Peyron C, Petit JM, Rampon C, Jouvet M, Luppi PH (1998). Forebrain afferents to the rat dorsal raphe nucleus demonstrated by retrograde and anterograde tracing methods. Neuroscience 82: 443-468.

Pham K, Nacher J, Hof PR, McEwen BS (2003). Repeated restraint stress suppresses neurogenesis and induces biphasic PSA-NCAM expression in the adult rat dentate gyrus. Eur J Neurosci 17: 879-886.

Pompeiano M, Palacios JM, Mengod G (1992). Distribution and cellular localization of mRNA coding for 5-HT1A receptor in the rat brain: correlation with receptor binding. J Neurosci 12: 440-453.

Puig MV, Santana N, Celada P, Mengod G, Artigas F (2004). In vivo excitation of GABA interneurons in the medial prefrontal cortex through 5-HT3 receptors. Cereb Cortex 14: 1365-1375.

Radley JJ, Rocher AB, Miller M, Janssen WG, Liston C, Hof PR et al (2005). Repeated stress induces dendritic spine loss in the rat medial prefrontal cortex. Cereb Cortex 3: 313-320.

Radley JJ, Sisti HM, Hao J, Rocher AB, McCall T, Hof PR et al (2004). Chronic behavioral stress induces apical dendritic reorganization in pyramidal neurons of the medial prefrontal cortex. Neuroscience 125: 1-6.

Rajkowska G, Miguel-Hidalgo JJ, Wei J, Dilley G, Pittman SD, Meltzer HY et al (1999). Morphometric evidence for neuronal and glial prefrontal cell pathology in major depression. Biol Psychiatry 45: 1085-1098.

Rapp S, Baader M, Hu M, Jennen-Steinmetz C, Henn FA, Thome J (2004). Differential regulation of synaptic vesicle proteins by antidepressant drugs. Pharmacogenom J 4: 110-113.

Robinson TE, Kolb B (1999). Alterations in the morphology of dendrites and dendritic spines in the nucleus accumbens and prefrontal cortex following repeated treatment with amphetamine or cocaine. Eur J Neurosci 11: 1598-1604.

Rutishauser U (1996). Polysialic acid and the regulation of cell interactions. Curr Opin Cell Biol 8: 679-684.

Santana N, Bortolozzi A, Serrats J, Mengod G, Artigas F (2004). Expression of serotonin1A and serotonin2A receptors in pyramidal and GABAergic neurons of the rat prefrontal cortex. Cereb Cortex 14: 1100-1109.

Seki T, Arai Y (1991a). Expression of highly polysialylated NCAM in the neocortex and piriform cortex of the developing and the adult rat. Anat Embryol (Berlin) 184: 395-401.

Seki T, Arai Y (1991b). The persistent expression of a highly polysialylated NCAM in the dentate gyrus of the adult rat. Neurosci Res 12: 503-513.

Seki T, Rutishauser U (1998). Removal of polysialic acid-neural cell adhesion molecule induces aberrant mossy fiber innervation and ectopic synaptogenesis in the hippocampus. J Neurosci 18: 3757-3766.

Steinbusch HW (1981). Distribution of serotonin-immunoreactivity in the central nervous system of the rat-cell bodies and terminals. Neuroscience 6: 557-618.

Strakowski SM, DelBello MP, Adler C, Cecil DM, Sax KW (2000). Neuroimaging in bipolar disorder. Bipolar Disord 2: 148-164.

Takagishi M, Chiba T (1991). Efferent projections of the infralimbic (area 25) region of the medial prefrontal cortex in the rat: an anterograde tracer PHA-L study. Brain Res 566: 26-39.

Theodosis DT, Bonfanti L, Olive S, Rougon G, Poulain DA (1994). Adhesion molecules and structural plasticity of the adult hypothalamo-neurohypophysial system. Psychoneuroendocrinology 19: 455-462.

Torregrossa P, Buhl L, Bancila M, Durbec P, Schafer C, Schachner $M$ et al (2004). Selection of poly-alpha 2, 8-sialic acid mimotopes from a random phage peptide library and analysis of their bioactivity. J Biol Chem 279: 30707-30714.

Varea E, Blasco-Ibañez JM, Gomez-Climent MA, Castillo-Gomez E, Crespo C, Nacher J et al (2005). PSA-NCAM expression in rat medial prefrontal cortex. Neuroscience 136: 435-443. 
Vega E, Gomez-Villalobos MJ, Flores G (2004). Alteration in dendritic morphology of pyramidal neurons from the prefrontal cortex of rats with renovascular hypertension. Brain Res 1021: 112-118.

Vertes RP (2004). Differential projections of the infralimbic and prelimbic cortex in the rat. Synapse 51: 32-58.

Wellman CL (2001). Dendritic reorganization in pyramidal neurons in medial prefrontal cortex after chronic corticosterone administration. J Neurobiol 49: 245-253.

West MJ (1993). New stereological methods for counting neurons. Neurobiol Aging 14: 275-285.
Willins DL, Deutch AY, Roth BL (1997). Serotonin 5-HT2A receptors are expressed on pyramidal cells and interneurons in the rat cortex. Synapse 27: 79-82.

Wong DT, Horng JS, Bymaster FP, Hauser KL, Molloy BB (1974). A selective inhibitor of serotonin uptake: Lilly $110140,3-(p-$ trifluoromethylphenoxy)- $\mathrm{N}$-methyl-3-phenylpropylamine. Life Sci 15: 471-479.

Zhang H, Miller RH, Rutishauser U (1992). Polysialic acid is required for optimal growth of axons on a neuronal substrate. J Neurosci 12: 3107-3114. 